\title{
Erratum to: Enhanced Color Image Method for Watermarking in RGB Space Using LSB Substitution
}

\author{
Mouna Bouchane, Mohamed Tarhda and Laamari Hlou
}

Erratum to:

"Enhanced Color Image Method for Watermarking in RGB Space Using LSB Substitution" in:

A. El Oualkadi et al. (eds.), Proceedings of the Mediterranean Conference on Information \& Communication Technologies 2015, Lecture Notes in Electrical Engineering 380, DOI 10.1007/978-3-319-30301-7_17

The spelling of the author's name was incorrectly given as "Mohamed Taghda" instead of "Mohamed Tarhda" in the book post-publication. The erratum chapter and the book have been updated with the change.

The updated original online version for this chapter can be found at 10.1007/978-3-319-30301-7_17

\footnotetext{
M. Bouchane $(\bowtie) \cdot$ M. Tarhda $\cdot$ L. Hlou

Electrical Engineering and Energy Systems Laboratory, Ibn Tofail University, BP. 133, Kenitra, Morocco

e-mail: mouna.bouchane@gmail.com

M. Tarhda

e-mail: tarhdamo@yahoo.fr

L. Hlou

e-mail: hloul@yahoo.com 